\title{
Estudio de las estrategias para mitigar las venas abiertas fruto de la extracción de la roca caliza en el departamento del Cesar
}

Martha Lucía Mendoza Castro', Johana Cecilia Peláez Aguirre²

\section{Resumen}

El presente trabajo de investigación consiste en el estudio de las estrategias para mitigar los impactos ambientales fruto de la extracción de la roca caliza en el departamento del Cesar. Esta investigación tiene como objeto de estudio las canteras de caliza del departamento del Cesar y estuvo delimitada a las que se encuentran en la etapa más productiva en la extracción de la valiosa roca. La investigación se realizó con base en la metodología descriptiva de tipo cuantitativa, obteniendo información de diversas fuentes, como documentos de bases de datos, encuestas, observaciones y una revisión de literatura. Se analizan dos variables a lo largo de la investigación como lo son las venas abiertas y la roca caliza. En esta investigación se identificaron las canteras dedicadas a la extracción de la roca caliza y, a su vez, se evaluó el impacto que esta trae consigo, logrando con esto el diseño de un plan de mitigación, el cual puede utilizarse para reducir los estragos que deja al medio ambiente (flora, fauna, suelo, agua, paisaje, aire, etc.) la minería. La evaluación ambiental realizada en este trabajo concluyó que las actividades que más impactan al medio ambiente se resumen en la etapa de construcción y montaje y la extracción de la roca, en las cuales hay una alteración física del medio circundante en la que está depositada.

Palabras clave: venas abiertas, roca caliza, canteras, impactos ambientales.

\footnotetext{
${ }^{1}$ Administradora de empresas, docente del programa Ingeniería de Minas de la Fundación Universitaria del Área Andina sede Valledupar. Correo: mmendoza27Rareandina.edu.co

${ }^{2}$ Estudiante de Ingeniería de Minas de la Fundación Universitaria del Área Andina sede Valledupar. Correo: jpelaez10Restudiantes. areandina.edu.co
} 


\section{Introducción}

La minería ha sido una de las actividades más antiguas y representa una de las fuentes de mayor inversión en nuestro país, por ende, es un beneficio para la economía; por esta razón, el departamento del Cesar se ha convertido en una de las víctimas de la práctica desmedida de la explotación de canteras; entre los minerales que se explotan se destacan arena, grava, arcilla, rocas ornamentales, barita $\mathrm{y}$, principalmente, caliza. La roca caliza es un mineral de origen sedimentario, el cual se forma a partir de la acumulación $y$ compactación de diversas materias, tales como restos calcáreos de seres vivos; es de gran importancia porque, a partir de su obtención, se puede utilizar para la construcción, productos químicos, agroquímicos y el vidrio.

La minería es una de las actividades más importantes en la economía cesarense, la cual se ha visto afectada de manera positiva y negativa, positiva, porque es una fuente de empleo para la mayoría de los habitantes de la parte centro del departamento; negativa porque genera un impacto ambiental muy grande en dicha zona. Referente a su estructura, pasó de tener una alta participación en el algodón a un alza en la minería. La minería es una gran alternativa para el mejoramiento económico, pero acarrea un sin número de consecuencias sociales y ambientales que dañan la integridad de la sociedad en la que se desarrolla dicha actividad, como los problemas que se han ido afianzando con la sociedad que genera la misma extracción de la roca caliza.

Toda extracción de mineral trae consigo una serie de efectos secundarios que son los que afectan la capa vegetal en la que el ser humano desarrolla sus actividades cotidianamente, y tales daños pueden llevar a la destrucción de los suelos naturales en los que se presentan limitaciones físicas, químicas y biológicas que dificultan el libre desarrollo de la naturaleza; no solo se tiene la afectación de la capa vegetal, sino la contaminación de las vertientes hídricas que constituyen el subsuelo y, por supuesto, sin pasar por alto las partículas que se disipan en el aire.

La presente investigación es importante porque se centra en el estudio de los impactos generados por la extracción de la roca caliza en el departamento del Cesar, la cual genera unas venas abiertas y se hace necesario una evaluación ambiental, ya que es una herramienta para estimar el cambio o modificación 
de los componentes abióticos, bióticos, socioeconómico y cultural existentes en un área definida, como consecuencia de la ejecución de actividades antrópicas o de un proyecto, en función de las condiciones previas a la acción de un proyecto, conforme a la normatividad vigente y aplicable.

En este sentido, la investigación sobre el problema señalado se considera importante debido a que permite estudiar las estrategias para mitigar las venas abiertas fruto de la extracción de la roca caliza en el departamento del Cesar. En este contexto, la investigación, al identificar los factores determinantes de las venas abiertas fruto de la extracción de la roca caliza, puede intentar explicar cuáles son los factores que influyen directamente en que hayan o no estrategias que mitiguen los impactos en dichas canteras.

Por otra parte, este estudio aportará un valor práctico permitiendo a los dueños de canteras y de próximas concesiones hacer una aplicación de las estrategias de mitigación incumbidas por la extracción de la roca caliza. Por consiguiente, los resultados de esta investigación permitirán generar nociones científicas, las cuales asientan establecer la importancia de los impactos ambientales desde la teoría de la extracción de la roca caliza para generar las innovaciones necesarias asociadas a las actividades principales del proceso de extracción de la roca.

En este sentido, se observará un aporte social en la investigación, la cual es justificada por los resultados obtenidos de la variable impacto como estrategia para disminuir las venas abiertas generadas por la extracción de la roca caliza en el departamento del Cesar, para que a partir de esto se genere crecimiento en los empleos con beneficios derivados de la investigación que apoya el mejoramiento del ecosistema.

Seguidamente, otro aporte es el metodológico, el cual se realiza con las variables en estudio venas abiertas de la extracción de la roca caliza, una de las pocas que aplica este método; por lo tanto, es base para otras investigaciones como estudios que evalúen, por ejemplo, la aplicación de las estrategias de mitigación para la extracción de la roca caliza, a fin de elevar la productividad y competitividad.

En otro contexto, al finalizar el estudio el mismo pretende dar a conocer la importancia de crear un conjunto de estrategias de mitigación para mantener la estabilidad de los ecosistemas, para 
cuando se realice extracción de la roca caliza en el departamento del Cesar.

Basado en los escenarios expuestos, se realizó esta investigación con el fin de dar una nueva visión sobre la manera de mitigar los impactos que generan las canteras extractoras de la roca caliza en el departamento del Cesar, siendo esta base teórica de suma importancia para el desarrollo de unas estrategias que ayuden a la disminución de las venas abiertas fruto de la extracción de la roca caliza, buscando el desarrollo de las canteras del departamento del Cesar y sus alrededores.

\section{Materiales y métodos}

En esta investigación se aplicó un estudio con enfoque mixto de tipo descriptivo; ya que en este tipo de estudios "se selecciona una serie de cuestiones y se mide cada una de ellas independientemente, de forma tal de describir los que se investiga, este tipo de estudio ofrece la posibilidad de llevar a cabo algún nivel de predicción; además son cuantificables los datos obtenidos" (Cauas).

La metodología adoptada para la realización del presente trabajo se fundamenta en el reconocimiento e identificación de las canteras de caliza dedi- cadas a la extracción de esta roca en el departamento del Cesar, de la misma manera se emplean recursos para la valoración sobre el impacto generado fruto de la explotación desmedida de dicha roca, teniendo en cuenta todas las recomendaciones teóricas para el diseño de un plan de mitigación con base en los daños provenientes de la extracción de la roca caliza en el Cesar.

\section{Población de estudio}

Según Levin y Rubin (1999, p. 135), una población "es el conjunto de todos los elementos que se estudian y acerca de los cuales se intenta sacar conclusiones".

Por su parte, según Hernández et al. (2006, p. 562) asegura que "la muestra en el proceso cualitativo es un grupo de personas, eventos, sucesos, comunidades, etc., sobre el cual se habrán de recolectar los datos, sin que necesariamente sea representativo del universo o población que se estudia".

Población. 35 canteras, las cuales corresponden a todas las canteras de la roca caliza en el departamento del Cesar.

Muestra. El estudio se realizó sobre una muestra de siete canteras dedicadas a la extracción de la roca caliza en el departamento del Cesar. 


\section{Instrumentos}

Al ser una investigación mixta de tipo descriptiva, se hizo necesaria la aplicación del instrumento recolector de datos, llamado encuestas para proceder a hacer la valoración de los impactos generados por la extracción de la roca caliza en el departamento del Cesar, utilizando a su vez la matriz de Leopold, en la que se reflejaron los resultados obtenidos una vez se hicieron las respectivas visitas de campo a cada una de las canteras.

\section{Resultados}

De acuerdo con la información suministrada y recolectada por la Agencia Nacional de Minería (ANM), de los resultados obtenidos se lograron identificar un total de 34 canteras dedicas a la extracción de la roca caliza en el departamento del Cesar (ver figura 1), de las cuales 17 se encuentran en etapa de exploración, cinco en construcción y montaje; mientras que las doce restantes están en la etapa más productiva de la minería que es la extracción, producción y comercialización de esta valiosa y apreciada roca (ver figura 2).

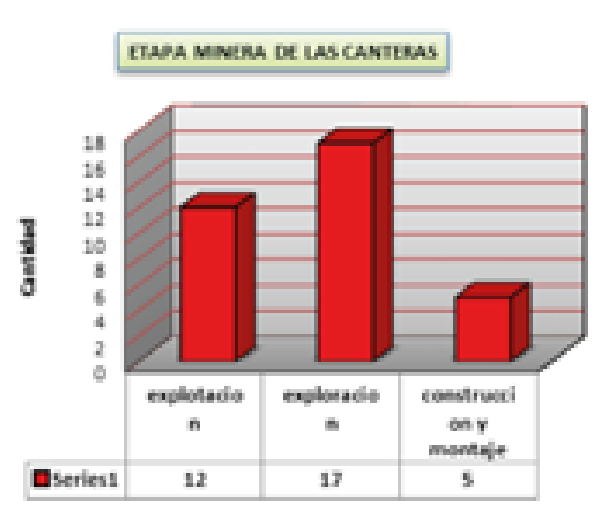

Figura 1. Etapa mineras de las canteras del departamento del Cesar; son de suma importancia para la investigacion las que se encuentran en etapa de explotacion

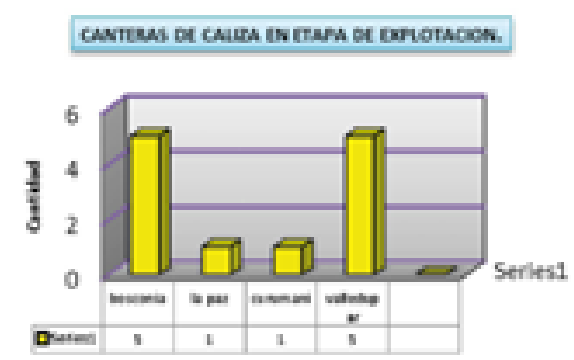

Figura 2. Ubicación de las canteras de caliza en etapa de explotacion en el departamento del Cesar

Para la presente investigación se delimitó una muestra de trece canteras de caliza en el departamento del Cesar; para las cuales solo se le aplicó la respectiva encuesta a siete de estas debido a que las seis restantes se encontraban 
cerradas o no estaban en su etapa más productiva. Por consiguiente, con toda la información adquirida en campo, se logró realizar el estudio de los impactos ambientales ocasionados por la extracción de la roca caliza en el departamento del Cesar.

Después de realizada la investigación y aplicación de la matriz de Leopold modificada, se observó que hay recursos naturales más afectados que otros y que existen actividades en los proyectos mineros que generan más impactos ambientales que otras, para los cuales se denotan de la siguiente manera:

Componentes naturales. En primera instancia, se encontró el componente atmosférico, en cuanto a lo que respecta al deterioro de la calidad del aire por la generación de material particulado y gases emitidos en cada una de las actividades mineras, presenta los valores más altos negativos (genera los mayores impactos ambientales); y esto es debido, principalmente, por la constante remoción de masas, vehículos traficando, arranque y transporte de este; también existe un incremento de los niveles de ruido producto de estos mismos procesos en la actividad minera.
Por otro lado, esta actividad minera de extracción de la roca caliza conlleva un deterioro de la calidad de los suelos en cuanto a su remoción y pérdida de estos por la no disposición de áreas especiales para su respectivo cuidado, que conlleva a que se den procesos de erosión de estos suelos (eólica e hídrica) $\mathrm{y}$ es debido, principalmente, a que una vez descapotada el área de trabajo estos suelos quedan desprovistos de protección y es entonces cuando los agentes atmosféricos, especialmente la lluvia, impactan sobre esta área descubierta y rebasa con la poca capa de suelo preexistente.

De igual modo, dentro los componentes físicos se destaca el paisaje; la actividad minera tiene mucha incidencia en este debido a la alteración del paisaje, se da principalmente debido a la extracción masiva que se hace de la roca caliza en el departamento del Cesar, dejando, a su vez, gigantescas venas o cráteres que le suman importancia para la determinación de alternativas en este punto de la investigación.

Ahora bien, la eliminación de la capa vegetal es de suma importancia, puesto que en esta se encuentran los nutrientes (ciclo de nutrientes) necesarios para el desarrollo de la fauna y flora existente 
en la zona; pero, cuando se inician labores de minería, lo primero que se hace es un descapote o retiro de esta capa para lograr acceder al mineral, sin desmeritar que en la mayoría de los casos existe una pérdida de dicha cobertura vegetal por falta de un espacio para la efectiva disposición de esta.

Cabe concluir que la actividad minera genera impactos de gran importancia en los componentes bióticos y abióticos. En la parte social existen afectaciones de la salud, principalmente por los niveles de material particulado que se genera a lo largo de toda la actividad, pero para los casos expuestos en el departamento del Cesar la población se encuentra bastante alejada de la zona donde se llevan a cabo estas labores y, por ende, debe hacerse precaución. Cabe señalar que en el componente económico existe un crecimiento exponencial desde el inicio de las operaciones; ya que esta genera expectativas de empleos para los pobladores de las zonas aledañas y así a contribuir al desarrollo de estas.

\section{Actividades mineras. Los} componentes naturales y las actividades mineras se encuentran correlacionadas de tal forma que para que una se cause necesita de otra que la produzca, esto quiere decir que las afectaciones a los componentes bióticos, abióticos y socioeconómicos son producto directamente de las actividades mineras llevadas a cabo para la extracción de la roca caliza en el departamento del Cesar. De este modo, y con base en lo analizado en campo y expuesto en la matriz de Leopold modificada, se logró analizar que la actividad que mayor impacto está generando es la construcción, montaje y adecuación de edificaciones y obras civiles, y es causado principalmente porque en esta etapa es en la que se realizan las mayores modificaciones biótica y abióticas.

Paralelamente a esto, la remoción de cobertura vegetal que se necesita hacer para la extracción del recurso natural lleva consigo a que sea una actividad que genere grandes impactos porque degrada y minimiza el área en la que se desarrolla la vida silvestre y, en consecuencia, hay una reducción y pérdida de biodiversidad de la zona. Mientras tanto, la adecuación de vías de acceso y la movilización y operación de equipos son actividades constantes que generan gran polución y deterioro de la cobertura vegetal, incidiendo drásticamente sobre la zona en la que se lleva a cabo las labores mineras. 
En cuanto a las actividades mineras que generan los suficientes trastornos en el medio, se encuentran aquellas que son llevadas a cabo durante el arranque, cargue y transporte de material, ya que estas tienen la particularidad de alterar la calidad de los suelos, pérdidas de propiedades (principalmente físicas) de estos, alteración de paisajes, contacto directo con la fauna y la flora, polución, entre otras que van sumándole a los impactos que genera el aprovechamiento de la roca caliza.

Después de realizada la investigación, de aplicar la matriz de Leopold modificada y de analizar los respectivos impactos causados por la extracción de la roca caliza, se llegó al punto más importante de la investigación, que es la elaboración de estrategias que logren mitigar las venas abiertas (impactos ambientales) fruto de la extracción de la roca caliza en el departamento del Cesar. En el análisis de la matriz de Leopold se evidenció de que, según el medio, existen recursos naturales más afectados que otros, por consiguiente, se denotan de la siguiente manera en el gráfico:

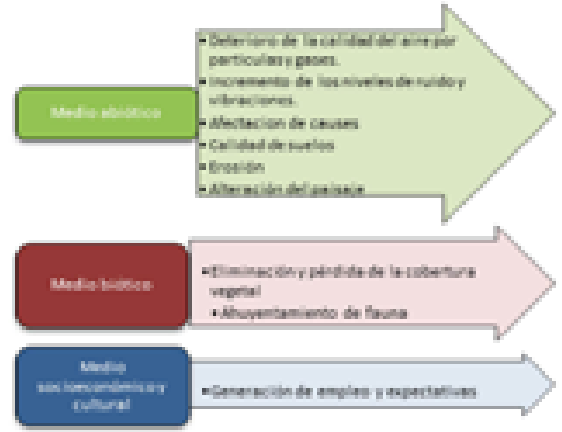

Fuente: elaboración propia.

Con respecto al grafico anterior, se designan una serie de estrategias influyentes con las cuales se espera mitigar los impactos ambientales causados por la extracción de la roca caliza en el departamento del Cesar. Dichas estrategias se contemplan a continuación, las cuales resumen los objetivos establecidos para dar con el cumplimiento de los compromisos ambientales que deben llevar a cabo las canteras en estudio; esto con el fin de establecer medidas y acciones compensatorias, evitar al máximo efectos contaminantes, promover la gestión ambiental, prevenir y reducir al máximo las afectaciones generadas por la extracción de la roca caliza.

Todo lo anterior debe ser implementado por las canteras extractoras de la roca caliza y darles un respectivo monitoreo y seguimiento 
en pro de verificar el cumplimiento y efectividad de dichas estrategias de mitigación de los impactos ambientales causados por la extracción de la roca caliza en el departamento del Cesar.

\section{Conclusión}

Se ha podido identificar cuáles son aquellas canteras dedicadas a la extracción de roca caliza en el departamento del Cesar, las cuales han hecho una extracción desmedida en las zonas de influencia, para ello se han identificado y encuestado para su posterior utilización, que son la base esencial para el desarrollo de los demás objetivos planteados en esta investigación.

Mientras tanto, la evaluación ambiental realizada en este trabajo nos arrojó como deducción que las actividades que más impactan al medio ambiente se resumen en la etapa de construcción y montaje y la extracción de la roca, en las cuales hay una alteración física del medio circundante en el que está depositada esta. Con base en estas actividades, también se determinó que los componentes más afectados son los siguientes: la calidad del aire, el paisaje, la morfología de los suelos y la reducción de flora y fauna, particularmente a estos componentes se les afecta negativamente; pero desde el punto de vista positivo tenemos la generación de empleo y expectativas por parte de los pobladores aledaños al proyecto minero.

No obstante, el levantamiento de la evaluación ambiental se realizó de forma cualitativa y cuantitativa, esto nos permite identificar los diferentes elementos ambientales que son susceptibles a sufrir cambios o, en su defecto, de las actividades impactantes que se ejerzan sobre estos elementos; sin embargo, vale la pena recalcar que se necesitan de otros criterios para analizar más allá de la matriz de Leopold los impactos que se lleguen a manifestar de forma indirecta a las zonas en estudio.

De otro modo, para lograr el equilibrio en la minería necesitamos de la correcta vigilancia y fiscalización de las autoridades ambientales y mineras, en las que las empresas mineras cuenten con un acompañamiento técnico para el desarrollo de sus labores, con el fin de controlar los procesos extractivos de este $u$ otros minerales que conllevan a un daño al medio ambiente, de forma que se practique y se logre la minería responsable. 
En justa medida, es necesaria la correcta implementación de los PMA que se llevan a cabo por los entes fiscalizadores, ya que estos son quienes tienen las medidas necesarias de prevención, corrección, mitigación y compensación; y es entonces cuando tiene valor agregado la investigación, ya que estas estrategias pueden ser incluidas dentro de estos planes, programas o proyectos para su correcta aplicación y verificación en la mitigación de impactos ambientales causados, en este caso, por la roca caliza. Sim embargo, también es necesario mencionar la adquisición de conocimiento de las revisiones documentales para poder diseñar este estudio.

Entonces, el municipio de Valledupar, Cesar es un gran productor de la roca caliza, lo cual genera una extracción desmedida de este recurso, creando, a su vez, un sin número de impactos en los componentes bióticos, abióticos y socioeconómicos, determinados a partir de la evaluación en la matriz de Leopold modificada y que anteriormente fue expuesta en los resultados. Con dichos resultados obtuvimos una serie de estrategias que buscan mitigar las venas abiertas fruto de la extracción de la roca caliza en el departamento del Cesar para las empresas productoras de esta roca y que, a su vez, también estas sean un referente para otros recursos naturales no renovables existentes a nivel nacional e internacional para el mejoramiento de la industria minera por medio de una minería ambientalmente responsable, fiscalmente sostenible y socialmente solidaria.

\section{Referencias}

Cauas, D. Definición de las variables, enfoque y tipo de investigación.

Hernández, R., Fernández, C. y Baptista, P. (2006). Metodología de la investigación, 4. ${ }^{\mathrm{a}}$ ed. México: McGraw Hill.

Levin, R. y Rubin, D. (1996). Estadística para administradores. México: Prentice Hall. 